\title{
Medical Microbiology
}

CASE REPORT

\section{Streptococcus salivarius Meningitis in Immunocompetent: A Case Report}

\author{
Abdelrahman M Elsawy ${ }^{1 *}$, Hani S Faidah ${ }^{2}$ and Elrashdy M Redwan ${ }^{3}$ \\ ${ }^{1}$ Department of Microbiology, Al-Azhar Faculty of Medicine, Egypt \\ ${ }^{2}$ Department of Microbiology, Umm Al-Qura University, Saudi Arabia \\ ${ }^{3}$ Biological Science Department, King Abdulaziz University, Saudi Arabia
}

*Corresponding author: Elsawy AM, Department of Microbiology, Al-Azhar Faculty of Medicine, Egypt; P.O. Box 13765, Makkah 21599, Saudi Arabia, Tel: 00966555-38337, E-mail: alsawy@hotmail.com

\begin{abstract}
Streptococcus salivarius (S. salivarius) is a rare cause of purulent meningitis. We report a case of meningitis due to $S$. salivarius in 15-years-old Sudanese boy. Diagnosis was established based on Cerebrospinal fluid (CSF) findings. The patient responded well to systemic antibiotics and recovered completely without any neurological complications after proper supportive measures. In this patient the meningitis caused by $S$. salivarius was thought to be spontaneous. The importance of bacteriological diagnosis and proper antibiotic treatment for $S$. salivarius meningitis is discussed.
\end{abstract}

\section{Keywords}

Streptococcus salivarius, Meningitis, Immunocompetent, Cerebrospinal fluid

\section{Introduction}

Streptococcus salivarius is a viridans group streptococcus found as normal flora in the human oral cavity [1]. Where it is the predominant species cultivated from tongue dorsa, and has been used as a reliable marker for forensic identification of saliva using DNA amplification techniques [2]. It is frequently considered a contaminant and is rarely the source of infection and meningitis due to Streptococcus salivarius species infections is relatively uncommon condition. More recent reports have suggested that $S$. salivarius is most commonly associated with meningitis [3]. Streptococcus salivarius meningitis has been reported after spinal anesthesia and myelogram procedures [4].

\section{Case Report}

A 15-years-old, Sudanese boy presented to the emergency department of tertiary care hospital, which is a Joint commission international (JCl) accredited, with a history of high grade of fever 2 days ago, intermittent, relieved by paracetamol, severe headache and generalized fatigability. On day of admission, he developed one episode of tonic clonic convulsion at home and three times in emergency room. He was intubated and admitted in ICU.

Assessment of vital signs on presentation revealed a temperature of $39.2{ }^{\circ} \mathrm{C}$, pulse of 112 beats per minute, and blood pressure of $88 / 50 \mathrm{mmHg}$. The physical examination revealed that the patient was toxic, and irritable. Cardiovascular and respiratory systems were normal. Laboratory investigations revealed hemoglobin $13.4 \mathrm{~g} / \mathrm{dl}$, total leukocyte count 26.400 cells per cubic millimeter. Liver and renal function, serum electrolytes, calcium and magnesium were within normal limits.

The patient have no history of epidural anesthesia, spinal myelography, post-traumatic cerebrospinal fluid leaks, brain abscesses, pericranial fistulas or other procedures associated with S. salivarius contamination and also have no recent contact with meningitis patients.

In view of suspected central nervous system infection, lumbar puncture was done. Cerebrospinal fluid (CSF) analysis showed an elevated protein level of 169 $\mathrm{mg} / \mathrm{dl}$ (reference range, 15 to 45 ) and decrease glucose concentration of $5.8 \mathrm{mg} / \mathrm{dl}$ (reference range of 40 to $70 \mathrm{mg} / \mathrm{dl}$ ). Gram stain of CSF showed no bacteria, but white blood cells were increased 16 cells $/ \mathrm{cm}$, with $56 \%$ neutrophils and $44 \%$ lymphocytes. With the conclusion of pyogenic meningitis, patient was started on intravenous ceftriaxone $(2 \mathrm{gm} / 12 \mathrm{~h})$, vancomycin $(1 \mathrm{gm} / 12 \mathrm{~h})$

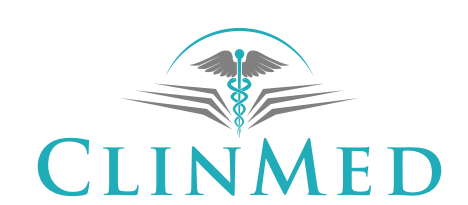

INTERNATIONAL LIBRARY

Citation: Elsawy AM, Faidah HS, Redwan EM (2018) Streptococcus salivarius Meningitis in Immunocompetent: A Case Report. Int Arch Med Microbiol 1:004.

Accepted: August 06, 2018; Published: August 08, 2018

Copyright: (C) 2018 Elsawy AM, et al. This is an open-access article distributed under the terms of the Creative Commons Attribution License, which permits unrestricted use, distribution, and reproduction in any medium, provided the original author and source are credited. 
and acyclovir $(750 \mathrm{mg} / 8 \mathrm{~h})$. The patient became a febrile within 48 hours of starting medications.

A Non-contrast computed tomography (CT) angiography of the brain showed the dural venous sinuses is mildly dilated with well opacification with no filling defects. The anterior and posterior circulations are well opacified with no filling defects or aneurysmal dilatation. In addition a non-contrast (CT) to paranasal sinuses showed mild focal mucosal thickening at both maxillary sinuses with intact walls of the paranasal sinuses.

The patient serum was negative to any viral markers for Herpes simplex virus 1 and 2, Varicella zoster virus, Epstein-Barr virus, HIV, Influenza, Mumps, Measles, Cytomegalovirus.

\section{Bacteria Identification}

Routine bacterial cultures of blood, sputum, urine and stool were negative, and on hospital day 3 a positive cultures were reported from CSF which was collected on the first day of admission.

The CSF specimen was cultured in BACTEC FX blood culture bottles and was incubated using BACTEC FX system. Three days after initial incubation, a positive culture signal was noted and direct Gram stain revealed Gram positive cocci in pairs.

The isolated S. salivarius strain grew at $37{ }^{\circ} \mathrm{C}$ and produced $\alpha$-hemolytic zones on agar containing horse blood and formed characteristic sticky rice ball-like colonies on agar plates with sucrose due to synthesize of soluble fructan. Catalase and oxidase were negative. Bacteria identification and antibiotic susceptibilities were determined by automated microbiology system (Vitek2, bioMérieux, USA), the isolated S. salivarius strain fermented glucose, sucrose, maltose, raffinose, inulin, salicin, trehalose, and lactic acid and did not ferment glycerol, mannitol, sorbitol, xylose, and arabinose. It was sensitive to ampicillin and ceftriaxone.

S. salivarius strain was not considered as contaminant due to the complete aseptic precautions that were followed during all procedures of the lumbar puncture and CSF sample culture in BACTEC FX blood culture bottles, also; $S$. salivarius strains are not one of the common contaminant strains in our hospital and finally all contaminant strains in our laboratory usually grow within maximally 48 hours of incubation.

\section{Conclusion}

Acute bacterial meningitis remains an important cause of morbidity and mortality worldwide, despite advances in prophylactic vaccination and pharmacotherapy [5]. Bacterial meningitis can be a serious and devastating illness. If the disease is not recognized and properly treated, it can result in seizures, neurologic deficits, and death [6].

Acute bacterial meningitis mostly presents with fe- ver, neck stiffness, and neurological symptoms. Classic triad can be present in some patient. Multiple studies showed missing of one or more symptoms in acute bacterial meningitis. However, virtually all patients have at least one of the findings of the classic triad of fever, neck stiffness, and neurological symptoms [7]. Here we report a spontaneous case of S. salivarius, typically patients presented with signs and symptoms of classic meningitis when infected with $S$. salivarius meningitis. These include fever most commonly, then headache, nuchal rigidity, altered mental status, nausea and vomiting [1].

The diagnosis of acute bacterial meningitis is mostly clinical along with CSF findings. In the right clinical setting headache, neck stiffness, and unexplained fever are highly suggestive of bacterial meningitis. The usual CSF findings are white blood cell count of 1000 to 5000/ $\mu \mathrm{L}$ with the percentage of neutrophils usually greater than $80 \%$, protein $>200 \mathrm{mg} / \mathrm{dL}$, and glucose $<40 \mathrm{mg} /$ dL. However, there have been reports shown in multiple studies where bacterial meningitis has no CSF abnormalities $[8,9]$.

Non-pneumococcal viridans group streptococci, such as Streptococcus salivarius, are very rare and account for $0.3-2.4 \%$ of all cases of bacterial meningitis, but increasingly reported infection caused by $S$. salivarius. They are normal flora of the gut and oral cavity and are considered low-virulence organisms [10]. However cases of meningitis have been reported, typically after procedures involving the spinal column. Other cases have been reported involving post-traumatic cerebrospinal fluid leaks, brain abscesses, and pericranial fistulas [11]. As recently underscored by the Centers for Disease Control and Prevention, S. salivarius and other viridans group streptococci are the most frequent causes of bacterial meningitis following spinal procedures such as anesthesia, accounting for up to $60 \%$ of cases [12].

The reported treatments of $S$. salivarius meningitis typically included a beta lactam antibiotic, most often either a penicillin or cephalosporin. The next most common medication was vancomycin [13]. For uncomplicated meningitis, the usual duration of antimicrobial therapy is 10 to 14 days, the patient under report was treated by vancomycin which is recommended by the American academy of pediatrics [14] because of the emergence of cephalosporin-resistant pneumococci and it does not need to be continued if the organism is susceptible to penicillin or cephalosporins, with intravenous third-generation cephalosporin's (ceftriaxone); as empirical chemotherapy for meningitis because of high bactericidal activity with low minimum inhibitory concentrations, high cure rate $(>80 \%)$ and less mortality $(<10 \%)$, ceftriaxone has poor intracellular penetration and enter the intercellular compartment that contains engulfed bacteria only by diffusion. In such a case the intracellular ability of the antibiotics is dependent on the extracellular concentration achieved [15]. 
Early diagnosis of the $S$. salivarius meningitis, together with an appropriate choice of the antibiotic at the initial presentation, followed by their proper duration, and regular follow up till patients completely recover can improve the outcome significantly.

\section{Conflicts of Interest}

All contributing authors declare no conflicts of interest.

\section{References}

1. Ayman Rihawi, Glen Huang, Royce Miller (2015) A Case of Spontaneous Streptococcus Salivarius Meningitis. Immunology and Infectious Diseases 3: 16-18.

2. Shewmaker PL, Gertz RE Jr, Kim CY, De Fijter S, DiOrio M, et al. (2010) Streptococcus salivarius meningitis case strain traced to oral flora of anesthesiologist. J Clin Microbiol 48: 2589-2591.

3. Scott W Sinner, Allan R Tunkel (2015) Viridans Streptococci, Nutritionally Variant Streptococci, Groups C and G Streptococci, and Other Related Organisms. In: John E Bennett, Raphael Dolin, Martin J Blaser, Mandell, Douglas, Bennett's principles and practice of infectious diseases. $\left(8^{\text {th }}\right.$ edn), Elsevier Inc., Philadelphia, 3160.

4. Allan R Tunkel, Diederik van de Beek, Ws Michael Scheld (2015) Acute Meningitis. In: John E. Bennett, Raphael Dolin, Martin J Blaser, Mandell, Douglas, Bennett's principles and practice of infectious diseases. ( $8^{\text {th }}$ edn), Elsevier Inc., Philadelphia, 1463.

5. Wilson M, Martin R, Walk ST, Young C, Grossman S, et al. (2012) Clinical and laboratory features of Streptococcus salivarius meningitis: a case report and literature review. Clin Med Res 10: 15-25.
6. Muralidharan R, Mateen FJ, Rabinstein AA (2014) Outcome of fulminant bacterial meningitis in adult patients. Eur J Neurol 21: 447-453.

7. Arif Aladag M, Refik M, Halil Ozerol I, Tarim O (2007) Posttraumatic Streptococcus salivarius meningitis in a child. $\mathrm{Pe}-$ diatr Int 49: 112-114.

8. Douglas Swanson (2015) Meningitis. Pediatrics in Review 36: 514-526.

9. Owusu M, Nguah SB, Boaitey YA, Badu-Boateng E, Abubakret AR, et al. (2012) Aetiological agents of cerebrospinal meningitis: a retrospective study from a teaching hospital in Ghana. Ann Clin Microbiol Antimicrob 11: 28.

10. Mehmood H, Khan N, Ullah S, Ullah A, Marwat A (2018) A Rare Case of Sphingomonas paucimobilis Meningitis in the Absence of Cerebrospinal Fluid Pleocytosis. J Investig Med High Impact Case Rep 6.

11. Fishbein DB, Palmer DL, Porter KM, Reed WP (1981) Bacterial meningitis in the absence of CSF pleocytosis. Arch Intern Med 141: 1369-1372.

12. Ris J, Mancebo J, Domingo P, Cadafalch J, Sanchez JM (1985) Bacterial meningitis despite normal CSF findings. JAMA 254: 2893-2894.

13. Megarbane B, Casetta A, Esvant H, Marchal P, Axler O, et al. (2000) Streptococcus salivarius acute meningitis with latent petromastoiditis. Scand J Infect Dis 32: 322-323.

14. Carley NH (1992) Streptococcus salivarius bacteremia and meningitis following upper gastrointestinal endoscopy and cauterization for gastric bleeding. Clin Infect Dis 14: 947-948.

15. Centers for Disease Control and Prevention (CDC) (2010) Bacterial meningitis after intrapartum spinal anesthesia New York and Ohio, 2008-2009. MMWR Morb Mortal Wkly Rep 59: 65-69. 